\title{
Protection Level and Reusability of a Modified Full-Face Snorkel Mask as Alternative Personal Protective Equipment for Healthcare Workers during the COVID-19 Pandemic
}

\author{
Jean Schmitt, Lewis S. Jones, Elise A. Aeby, Christian Gloor, Berthold Moser, and Jing Wang*
}

Cite This: Chem. Res. Toxicol. 2021, 34, 110-118

Read Online

ACCESS | Llll Metrics \& More | 回 Article Recommendations | S1 Supporting Information

ABSTRACT: The worldwide outbreak of COVID-19 has drastically increased pressure on medical resources and highlighted the need for rapidly available, large-scale, and low-cost personal protective equipment (PPE). In this work, an alternative full-face mask is adapted from a modified snorkel mask to be used as PPE with two medical-grade filters and a $3 \mathrm{D}$-printed adapter. Since the mask covers the eyes, mouth, and nose, it acts as a full-face shield, providing additional protection to healthcare workers. The SARSCoV-2 has a size between $60 \mathrm{~nm}$ and $140 \mathrm{~nm}$, and airborne viral particles can be carried by larger droplets with sizes up to several millimeters. The minimum filtration efficiency of mechanical and electrostatic filters is usually reached between $30 \mathrm{~nm}$ and $300 \mathrm{~nm}$.

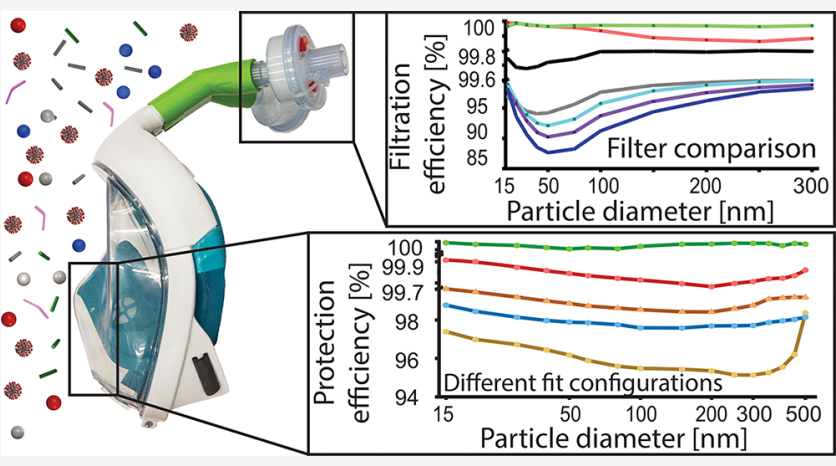
The filtration efficiency of different medical filters is measured for particles below $300 \mathrm{~nm}$ to cover the size of the SARS-CoV-2 and small virus-laden droplets, and determine the minimum efficiency. The filtration performance of the adapted full-face mask is characterized using $\mathrm{NaCl}$ particles below $500 \mathrm{~nm}$ and different fitting scenarios to determine the minimum protection efficiency. The mask is compared to a commercial respirator and characterized according to the EN 149 standard, demonstrating that the protection fulfills the requirements for the FFP2 level (filtering face-piece 2 , stopping at least $94 \%$ of airborne particles). The device shows a good resistance to several cycles of decontamination (autoclaving and ethanol immersion), is easy to be produced locally at low cost, and helps to address the shortage in FFP2 masks and face shields by providing adequate protection to healthcare workers against particles $<500 \mathrm{~nm}$ in size.

\section{INTRODUCTION}

The outbreak of COVID-19 in 2019 and the subsequent global spread through 2020 caused an overload of the healthcare system in many affected countries. Hospitals and medical facilities faced a shortage of personal protective equipment (PPE), highlighting the need for rapidly available and large-scale emergency solutions. ${ }^{1}$ With developing countries facing similar issues, low-cost and local availability are also important characteristics of the required PPE. Therefore, several research groups have worked on adapting and testing existing devices likely to be used as PPE, e.g., the use of charged nanofibers to filter SARS-CoV-2 ${ }^{2}$ and the successful adaptation of an elastomeric respirator as an alternative to the N95 respirators. ${ }^{3}$ Several projects are focusing on the filtration efficiency of a snorkel full-face mask modified with an open-source 3D-printed adapter. A study ${ }^{4}$ covered the filtration efficiency in the range of 0.3-10 $\mu \mathrm{m}$, together with the measurement of carbon dioxide accumulation and sound propagation through the mask. Another publication ${ }^{5}$ performed a successful fit test with a similar mask, fulfilling the OSHA N95 standard.

The SARS-CoV-2 has been measured between $60 \mathrm{~nm}$ and 140 $\mathrm{nm},{ }^{6}$ and aerosols containing SARS-CoV-2 are mostly found in the $250-500 \mathrm{~nm}$ size range. ${ }^{7}$ Particles below $10 \mu \mathrm{m}$ generated by a coughing patient are likely to travel over several meters before settling on the floor. ${ }^{8}$ A significant number of particles generated during expiratory activities are smaller than $800 \mathrm{~nm} .{ }^{9}$ The transmission of COVID-19 through virus-laden droplets is actively investigated. ${ }^{10,11}$ The most penetrating particle size (MPPS) corresponds to the size where the filters have their minimum efficiency and results from the combined effects of the filtration mechanisms (interception, inertial impaction, diffusion, and electrostatic attraction) at different particle sizes. The MPPS is usually in the range of $100-300 \mathrm{~nm}$ for mechanical filters and $30-70 \mathrm{~nm}$ for electrostatic filters. ${ }^{12}$ Therefore, measuring the penetration of particles with a diameter of $<500$ $\mathrm{nm}$ is critical since they are in the range where filters have their lowest efficiency, and may be highly contagious. This is because

Received: August 31, 2020

Published: December 17, 2020 
these particles exhibit high mobility and a long suspension time. The treatment of infected patients often involves aerosolgenerating medical procedures, such as intubation, bronchoscopy, and mechanical ventilation, and increases the generation of potentially contaminated droplets. ${ }^{13}$ The adapted full-face mask tested in this work prevents contaminated droplets from reaching the eyes, mouth and nose of medical staff performing such procedures. The adaptation of full-face snorkel masks with locally available filters and easy-to-produce adapters can constitute an alternative source of PPE to overcome the shortage in FFP2 masks and face shields.

The aim of the present work is to provide new data on modified snorkel masks, similar to those previously described, ${ }^{4,5}$ with a novel adapter designed to hold two medical filters. The focus is set on the size-resolved particle penetration below 500 $\mathrm{nm}$ as well as the influence of decontamination processes on the protection efficiency. In the first section, the filtration efficiency of several types of filters is measured and the quality factor is calculated based on filter pressure drop and filtration efficiency at the MPPS for particles between $12 \mathrm{~nm}$ and $300 \mathrm{~nm}$ in size. In the second part, the size-dependent particle penetration, from $15 \mathrm{~nm}$ to $500 \mathrm{~nm}$, in the full-face mask with the adapter and filters is measured while placed on a dummy head. Different fitting scenarios are considered and a commercial full-face respirator is tested as a reference. Finally, the impact of two decontamination methods (autoclaving and ethanol immersion) on the protection efficiency is measured.

\section{MATERIALS AND METHODS}

Filter Selection. The setup for the evaluation of the particle penetration in the filters was described and used in other research. ${ }^{12,14,15}$ Particles were generated from a solution of $\mathrm{NaCl}$ in deionized water by a single-jet atomizer (Model 3079A, TSI, Inc., USA). A $0.01 \mathrm{wt} \% \mathrm{NaCl}$ solution was used to generate particles smaller than $100 \mathrm{~nm}$ and a $1 \mathrm{wt} \%$ solution was used to generate particles equal to or larger than $100 \mathrm{~nm}$. The penetration was measured for particle sizes between $12 \mathrm{~nm}$ and $300 \mathrm{~nm}$. Although the smaller particle sizes were not relevant to evaluate the penetration of the SARS-CoV-2 (viral particles between $60 \mathrm{~nm}$ and $140 \mathrm{~nm}^{6}$ ), they were used here for two reasons: other viruses have smaller sizes (Picornaviruses between 22 $\mathrm{nm}$ and $30 \mathrm{~nm}$ in size, ${ }^{16}$ and Parvoviruses between $18 \mathrm{~nm}$ and $26 \mathrm{~nm}^{17}$ in size) and the characterization of the filters required the determination of the MPPS.

The polydisperse aerosol flow was set to $1 \mathrm{~L} / \mathrm{min}$ before entering the differential mobility analyzer (DMA, Model 3081, TSI, Inc., USA) working with a sheath flow of $10 \mathrm{~L} / \mathrm{min}$. The resulting monodisperse aerosol exited the DMA and the final flow rate was reached by mixing the aerosol with makeup air. Two condensation particles counters (CPC Model 3775, TSI, Inc., USA) sampled the air upstream and downstream of the filter. The measurements of the FFP2 and FFP3 filters were performed with a piece of the filtering material having a diameter of $50 \mathrm{~mm}$ mounted on a filter holder; the medical filters were measured as received (diameter of the filtering material: $55 \mathrm{~mm}$ ) and were directly connected to the tubing. The airflow downstream of the filter was treated by a HEPA filter and evacuated by the vacuum pump. The air stream was controlled by a mass flow controller (GFC37, Aalborg Instruments \& Controls Inc., USA) in order to maintain a constant face velocity of $8.5 \mathrm{~cm} / \mathrm{s}$ through the filtering material. It was decided to use a constant face velocity for all the filters in order to allow a direct comparison of their filtration efficiency. For the subsequent testing of the complete full-face mask, the face velocity was adapted to the chosen filter in order to reflect the operating conditions. The sampling time was $5 \mathrm{~min}$, with one measurement every second.

The filtration efficiency was calculated according to the following formula: ${ }^{18}$

$$
E=1-\frac{C_{\text {down }}}{C_{\text {up }}}
$$

where $C_{\text {down }}$ and $C_{\text {up }}$ represent the particle number concentration downstream and upstream from the filter.

The pressure drop was measured on the complete mask, with adapter and filters, installed on a polystyrene head. The setup is described in Figure S1 in the Supporting Information (pressure sensor: PX 409 Series, Omega Engineering, Inc., USA). The high pressure point was taken outside of the mask, between the two filters. The low pressure point was measured inside the lower chamber of the mask. The airflow through the mask was generated by two vacuum pumps and controlled by four flow controllers in order to reduce the pressure drop in the system. Flows of $65 \mathrm{~L} / \mathrm{min}$ and $95 \mathrm{~L} / \mathrm{min}$ were generated to represent the most challenging conditions met by the filters in a dual-filter configuration. As only one specimen was available for most of the filters, each one was alternatively installed on the left and the right inlet, the other one being sealed.

A seal check, adapted from the guidelines provided by a manufacturer of protective equipment, ${ }^{19}$ was performed before all the measurements:

- The airflow was set to $95 \mathrm{~L} / \mathrm{min}$

- The inlet of both filters was obstructed

- A significant movement of the mask toward the head should be observed, indicating an aspiration of the mask caused by the pressure drop, and, therefore, the absence of leakage

- If no movement was observed, the interface between the mask and the head was examined for potential leakages and the mask readjusted until the previous criteria was fulfilled.

Each measurement was repeated five times.

The quality factor was calculated using the following equation: ${ }^{20-22}$

$$
Q=\frac{-\ln (P)}{\Delta p}
$$

where $P$ is the particle penetration and $\Delta p$ is the pressure drop. The calculation was done by using the penetration at the MPPS and the pressure drop at $95 \mathrm{~L} / \mathrm{min}$ after subtracting the pressure drop generated by the mask without filters.

The tested filters were obtained from medical facilities and details are given in Table 1. Pictures of the filters are presented in Figure S2 in the Supporting Information.

Table 1. Information on the filters tested

\begin{tabular}{llll}
\multicolumn{1}{c}{ manufacturer } & \multicolumn{1}{c}{ name } & $\begin{array}{l}\text { filter } \\
\text { type }\end{array}$ & \multicolumn{1}{c}{ intended use } \\
UVEX & Silv-air C & FFP3 & face mask \\
HD Medis & - & KF94 & face mask \\
Dräger & TwinStar55 & HME & mechanical ventilation \\
Dräger & SafeStar55 & HEPA & mechanical ventilation \\
PharmaSystems & BACT HME Port & HME & mechanical ventilation \\
Intersurgical & Hydroguard mini & HEPA & mechanical ventilation \\
Intersurgical & Intertherm HMEF & HME & mechanical ventilation
\end{tabular}

Fabrication of the Adapters. The design of the adapter derives from the design of Filip Kober (printable ventilator-free respiratory: Subea Easybreath Mask Adapter, GrabCAD). Specifically, the single filter design was modified to incorporate two viral filters that filter both of the flow channels of the mask.

The part was printed in a vertical orientation (filter inlets positioned upward), using an Ultimaker 3 Extended using PLA, $0.4 \mathrm{~mm}$ nozzle diameter, $0.15 \mathrm{~mm}$ layer height, $20 \%$ infill and a skirt build plate adhesion (20 lines, $0 \mathrm{~mm}$ distance). PVA was used as support material, to support overhangs of $>45^{\circ}$.

Influence of the Chin Valve. The model of the snorkel mask used for all the measurements was an Easybreath (Decathlon). The chin valve was sealed with a laser-cut 3-mm-thick poly(methyl methacrylate) (PMMA) disk fused to the mask with acetone after the removal of the 
silicone valve. This sealing method follows several requirements to make it compatible with the intended use of the full-face mask:

(1) Inexpensive, simple, and fast, in order to be applied to large quantities of masks;

(2) Nontoxic, since it will be used inside a closed volume; and

(3) Robust, because it must withstand the various disinfection processes applied to the mask.

Further details on the other considered sealing methods are given in Figure S3 in the Supporting Information.

The particle penetration was measured in two steps: in the first step, the mask was fitted on the polystyrene head, in the second step, the mask was worn by a volunteer (whose informed consent was obtained) to follow a real breathing cycle. In both cases, the challenging aerosol was taken from the environment and two CPCs sampled the upstream and downstream aerosol. The measurements with the polystyrene head were performed at $65 \mathrm{~L} / \mathrm{min}$ and $95 \mathrm{~L} / \mathrm{min}$. The volunteer targeted a breathing frequency of 16 movements per minute. For the normal breathing, the functional residual capacity of the lungs was considered, while the inspiratory vital capacity was considered for the heavy breathing. Both volumes were calculated based on physiological parameters ${ }^{23}$ and led to a flow rate of $52 \mathrm{~L} / \mathrm{min}$ for normal breathing and $83 \mathrm{~L} / \mathrm{min}$ for heavy breathing.

The temperature and humidity values were acquired by four sensors (Model SHTC3, Sensirion AG, Switzerland). Sensors Nos. 1 and 2 were installed at each side of the upper chamber, No. 3 in the lower chamber, and No. 4 outside. The chin valve was tested both in the original configuration and completely sealed as described earlier. The mask was fitted on the volunteer and the seal check previously described was performed before each measurement.

Potential Leakage of the Adapter Assembly. The filters were assembled on the adapter and integrated in the setup previously described, the filter holder being replaced with the adapter. Minor adaptations were made to the setup: the penetration was measured for a polydisperse aerosol, without considering the different particle sizes and the challenging aerosol was taken from the environment rather than generated by the atomizer. The particle concentration and size distribution in the room where the measurement took place was considered as stable over the sampling time ( $5 \mathrm{~min}$ to complete an upstream and downstream measurement). Two filters SafeStar55 were mounted on the 3D-printed adapter. The upstream and downstream airflows were sampled by two CPCs at $1.5 \mathrm{~L} / \mathrm{min}$. The filtration efficiency was measured at both $65 \mathrm{~L} / \mathrm{min}$ and $95 \mathrm{~L} / \mathrm{min}$. Potential methods to improve the tightness were tested: winding of Teflon tape around the filter's inlet and sealing of the assembly by wrapping it in a piece of rubber.

Size-Dependent Penetration in the Full-Face Mask. The sizedependent particle penetration in the full-face mask was measured between $15 \mathrm{~nm}$ and $500 \mathrm{~nm}$. The maximum tested particle size was increased from $300 \mathrm{~nm}$ in the previous measurements to $500 \mathrm{~nm}$ in order to encompass the maximum penetrating particle size of the fullface mask. The minimum particle size was increased from $12 \mathrm{~nm}$ in the filter comparison to $15 \mathrm{~nm}$, because the dilution of the aerosol, resulting from the higher face velocity, drastically decreased the upstream concentration for $12 \mathrm{~nm}$ particles. The polydisperse aerosol stream was generated by a six-jet atomizer (Model 9306, TSI, Inc., USA) at an aerosol flow rate of $10 \mathrm{~L} / \mathrm{min}$ from a solution of $\mathrm{NaCl}$ in deionized water, using the same concentrations as previously mentioned. The aerosol flow was directly mixed with $20 \mathrm{~L} / \mathrm{min}$ filtered air in the atomizer. The flow passed through a diffusion dryer, a Kr-85 neutralizer, and was injected into a stainless steel chamber. At the entrance of the chamber, the aerosol was mixed again with filtered air. The airflow pumped out of the mask was set to $95 \mathrm{~L} / \mathrm{min}$, equivalent to heavy breathing. The sum of incoming airstreams (aerosol and makeup air) was set to be slightly higher than $95 \mathrm{~L} / \mathrm{min}$ in order to prevent external particles from entering the steel chamber and keep a low background concentration. The background concentration in the chamber was checked at the beginning of each measurement and was less than 1 particle $/ \mathrm{cm}^{3}$. The full-face mask was installed on a polystyrene head, and the particle concentration was measured from the lower chamber, using a sampling line running through the dummy head. The aerosol size distribution was measured by a scanning mobility particle sizer (SMPS, Model 3936L75, TSI, Inc., USA). The sampling time was set to $60 \mathrm{~s}$ for each particle size and the SMPS was controlled manually. The airflow of $95 \mathrm{~L} / \mathrm{min}$ was applied during the sampling of both upstream and downstream particle concentrations in order to generate the same pressure drop in both sampling lines and compensate for particle losses that were due to the high flow. The CPC operated under critical flow conditions and compensated the variations of the inlet pressure caused by the pressure drop in the face mask. The diameter and length of the tubing was adapted to minimize the pressure drop and keep the inlet pressure of the $\mathrm{CPC}$ in the range recommended by the manufacturer. Therefore, the influence of the pressure drop through the full-face mask on the inlet flow was negligible.

The commercial respirator used for comparison was a $3 \mathrm{M}$ Model 6800 system that was equipped with two filters, A2B2E2K2P3 R (P3 particle protection class according to EN 143).

The measurements according to the EN 149 standard were performed in an atmosphere charged with Paraffin aerosol. The aerosol concentration was measured with a Portacount Pro+ Respirator Fit Tester (Model 8038, TSI, Inc., USA) outside and inside the full-face mask while the volunteers performed a series of tasks (movement) described by EN 149:

While still walking, the subject performed the following exercises:

(1) walking for $2 \mathrm{~min}$ without head movement or talking;

(2) turning head from side to side ( $\sim 15$ times), as if inspecting the walls of a tunnel;

(3) moving the head up and down ( $\sim 15$ times), as if inspecting the roof and floor;

(4) reciting the alphabet or an agreed text out loud as if communicating with a colleague; and

(5) walking for $2 \mathrm{~min}$ without head movement or talking.

Decontamination Process. The decontamination cycles were performed on the mask only, without the adapter and the filters, as follows:

- Autoclaving: $22 \mathrm{~min}$ at $121^{\circ} \mathrm{C}(1.2 \mathrm{bar})$, followed by $30 \mathrm{~min}$ of drying.

- Ethanol immersion: $15 \mathrm{~h}$ immersion in a solution of $70 \mathrm{vol} \%$ ethanol in deionized water.

Both methods prove to be efficient to inactivate coronaviruses: a solution of $70 \%$ ethanol reduces the infectivity of two types of coronaviruses by $99.9 \%$ after $1 \mathrm{~min}^{24}$ Autoclaving is a widely used method to sterilize medical devices and a lower temperature of $70^{\circ} \mathrm{C}$ is already sufficient to inactivate SARS-CoV-2 within 5 min. ${ }^{25}$

Each mask was exposed to 10 cycles of one decontamination method. In order to have a stable aerosol concentration and size distribution throughout the entire duration of the measurements (several days), the challenging polydisperse aerosol was generated by a six-jet atomizer from a solution of $\mathrm{NaCl}$ in water concentrated at 0.01 and $1 \mathrm{wt} \%$. The size distributions of the generated particles were centered at $36 \mathrm{~nm}$ (0.01 wt \%) and $82 \mathrm{~nm}(1 \mathrm{wt} \%)$. The size distribution of the challenging aerosol was measured with an SMPS. The data are given in Figure S4 in the Supporting Information. The downstream and upstream concentrations were measured by a CPC while the sample was placed on the dummy head in the stainless steel chamber. The adapter and the filters were removed from the mask during the decontamination cycles. The exposure of filtration material to temperatures of $<100{ }^{\circ} \mathrm{C}$ does not impact their filtration efficiency and might therefore constitute an efficient way to inactivate SARSCoV-2 on the filters. ${ }^{26}$ However, using ethanol immersion to disinfect electrostatic filters might degrade their filtration performances through the loss of electrostatic charges. ${ }^{27}$

\section{RESULTS AND DISCUSSION}

Description of the Full-Face Mask and Adapter. Figures $1 \mathrm{a}-\mathrm{c}$ presents the full-face mask with the 3D-printed adapter and the two filters. 

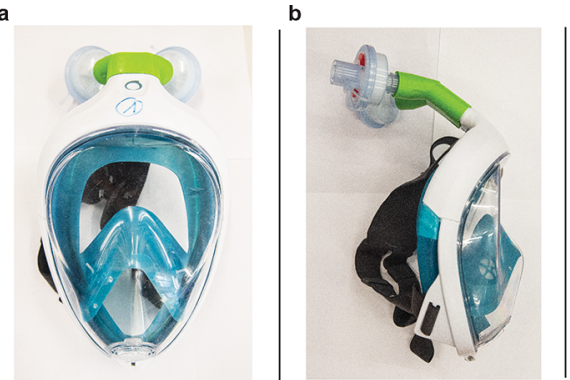

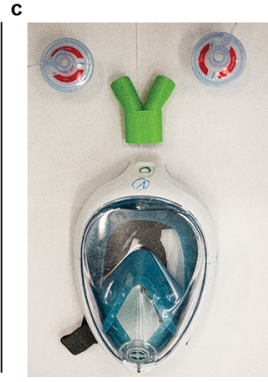

Figure 1. Pictures of the assembled full-face mask: (a) front view, (b) side view, and (c) expanded view showing the different components.

The details of the air pathways in the mask and through the adapter are given in Figure 2. The mask is divided into two

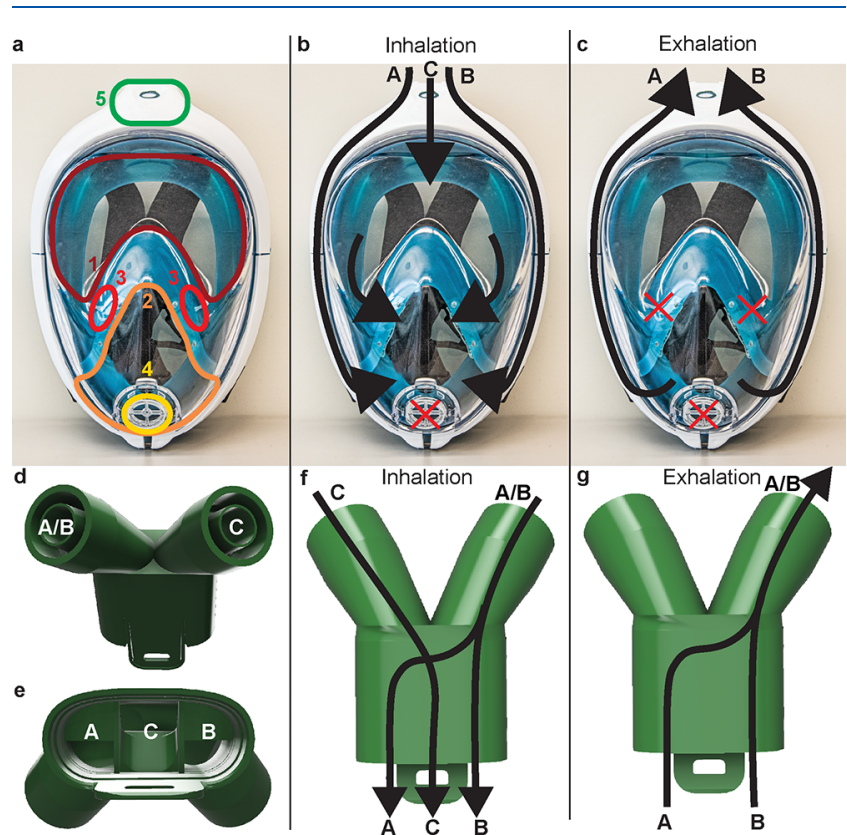

Figure 2. Snorkel mask and 3D-printed adapter. Panel (a) shows the subdivisions of the mask: the upper chamber (marked "1") encloses the eyes and lower chamber (marked " 2 ") covers the mouth and nose. They are separated by a piece of silicone holding two unidirectional valves (marked " 3 "). The lower chamber contains the unidirectional chin valve (marked "4"). The port for the adapter is located on the top (marked " 5 "). Panel (b) shows the airways during inhalation, as the air enters from all three inlets on top, marked "A", "B", and "C". Panel (c) shows the airways during exhalation (with a sealed chin valve) only involving the side channels, marked "A" and "B". Panels (d) and (e) show the 3D-printed adapter; the two medical filters are connected to the ports marked "A/B" and "C" in panel (d). The airflows through the adapter are shown in panel (f) for the inhalation and in panel (g) for the exhalation. The channels "A" and "B" merge into one channel (marked "A/B") inside the adapter.

chambers, with the upper one enclosing the eyes (marked as "1" in Figure 2a) and the lower one enclosing the mouth and nose (marked "2"). The chambers are separated by two unidirectional valves (marked " 3 "), which open during inhalation (Figure $2 b$ ) and close during exhalation (Figure 2c). The chin port (marked "4") also contains a unidirectional valve. Because of the potential expulsion of contaminated droplets with exhalation, the chin port was permanently sealed with a disk of PMMA. Sealing slightly reduced particle penetration during inhalation and did not appear to cause any increase in humidity or temperature.

Further details can be found in Figure S5 in the Supporting Information.

The 3D-printed adapter, shown in Figures $2 \mathrm{~d}-\mathrm{g}$, was adapted from an existing open-source design to enable the fitting of two filters. The filters and mask are connected by friction fit. The adapter was designed such that the attached filters are angled toward the back of the wearer's head; this provides protection against frontally projected airborne particles ${ }^{28}$ and is ergonomic. The adapter directs air inflow from the filters via a central channel (Figure $2 b$, marked "C") into the upper chamber of the mask, or via two lateral channels (Figure 2b, marked "A" and "B") into the lower chamber. This allows inhaled air to enter the mask through both filters, while exhaled air is expulsed only via the lateral channels of the mask and one filter. The one-way valves of the mask enable this flow separation, which reduces the breathing resistance of the assembly.

Tests under Real Operating Conditions. The full-face mask was tested for several days in a medical facility during the peak of the SARS-CoV-2 outbreak in Switzerland. It was worn for up to $16 \mathrm{~h}$ a day. An adapter with one filter (all three channels merged into one and led to one filter) was compared to the adapter used in this work. The breathing was reported to be easier with the two-filter adapter, leading to improved comfort over long periods of time. A seal check has been performed and the straps have been adapted before using the mask. The volunteers described a feeling of safety and protection brought by the full coverage of the face. The mask is also easy to handle with gloves, lowering the risk of contamination during the installation and removal of the full-face mask. However, the configuration of the face mask does not allow wearing glasses together with the mask.

Filter Selection. Different filters used for mechanical ventilation in intensive care units and operation rooms have been compared to each other and to FFP2 and FFP3 (respectively filtering face-piece " 2 " and " 3 ", corresponding to a filtration efficiency of at least $94 \%$ and $99 \%$, according to EN 149 standard) filtration materials taken from commercial respirators. The objective of the comparison was to select the filters with the highest efficiency for subsequent measurements: since the focus of this study was to characterize the full-face mask, the filter itself should have a limited influence on the measurements of the particle penetration into the mask. The experimental setup is presented in Figure 3a, and the particle penetration for each measured size at a constant face velocity of $8.5 \mathrm{~cm} / \mathrm{s}$ is given in Figure $3 \mathrm{~b}$.

The SafeStar55 and Hydroguard, both labeled as mechanical HEPA (high efficiency particulate air) filters, had the best filtration efficiency (>99.99\%). Their MPPS, which corresponds to the minimum filtration efficiency, was $\sim 250 \mathrm{~nm}$. The other filters, based on electrostatic filtration, had a smaller MPPS between $30 \mathrm{~nm}$ and $70 \mathrm{~nm}$, and the shape of the curve describing the filtration efficiency as a function of the particle size was similar to the FFP2 and FFP3 filter materials. Their efficiency was $>98 \%$ at $300 \mathrm{~nm}$. The filtration efficiency and MPPS measured for the FFP2 and FFP3 masks were similar to those measured elsewhere. ${ }^{29}$

The measurements of the pressure drop generated by the fullface mask equipped with the adapter and the filters are presented in Figures $4 a$ and $4 b$, together with the calculation of the quality factor in Figure 4c.

The pressure drop was lower for a flow of $65 \mathrm{~L} / \mathrm{min}$, compared to $95 \mathrm{~L} / \mathrm{min}$ for all filters and configurations, and the left inlet generated a lower pressure drop than the right one. This 


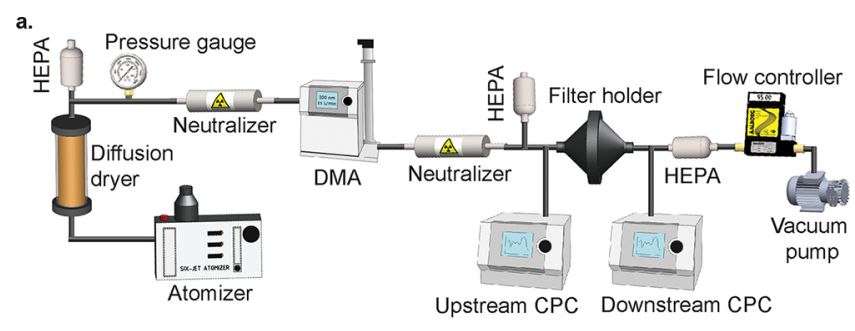

b.

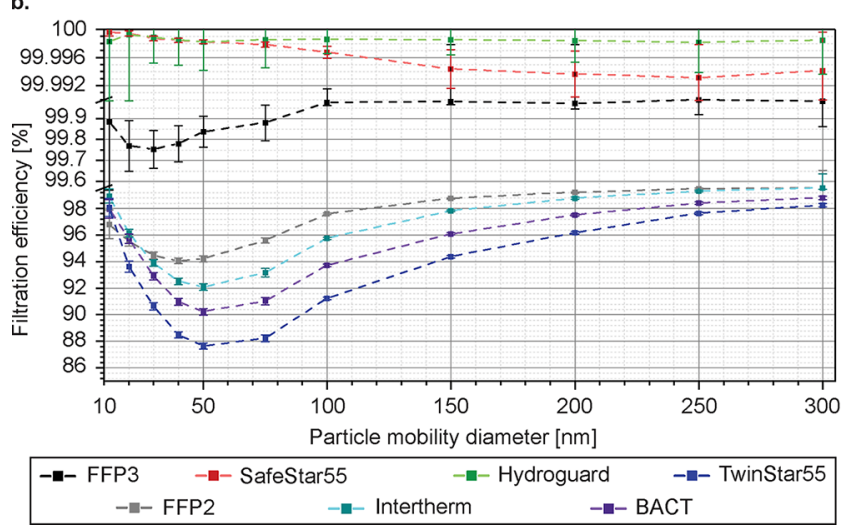

Figure 3. Measurement of the filtration efficiency of five medical grade filters and comparison with filtration media from FFP2 and FFP3 face masks. Panel (a) presents the experimental setup to measure the penetration of $\mathrm{NaCl}$ particles with different mobility diameters. The measurement was performed with a differential mobility analyzer (DMA) and two condensation particle counters (CPC). Panel (b) shows the filtration efficiency, as a function of the particle mobility diameter at a constant face velocity of $8.5 \mathrm{~cm} / \mathrm{s}$. The error bars refer to the standard deviation calculated from the 300 measurements points acquired during the 5-min samplings.

difference can be explained by the geometry of the full-face mask and the adapter: the left inlet was directly connected to the upper chamber and the flow resistance was lower, compared to that of the side channels of the mask, connecting the lower chamber to the right inlet. The use of the SafeStar55 in a dualinlet configuration (a filter connected to each port) significantly reduced the pressure drop, compared to the single-inlet configuration (a filter alternatively connected to the right and left port, the other one being sealed). The Hydroguard and SafeStar55 had significantly higher quality factors than the other filters. As expected, the dual-inlet configuration based on the SafeStar55 showed the highest quality factor as this configuration benefited from a significantly reduced pressure drop. Having a lower pressure drop than the SafeStar55, the Hydroguard would have displayed a higher quality factor in a dual-inlet configuration; however, because of filter availability, only the SafeStar55 was considered for the further measurements on the full-face mask. Scanning electron miscroscopy (SEM) images from the filtering material of the SafeStar55 filter are presented in Figure 5. The filter has multiple layers of fibers and a large variability in the diameters of the fibers, measured between $120 \mathrm{~nm}$ and $8 \mu \mathrm{m}$. Further images are presented in Figure S6 in the Supporting Information.

Size-Dependent Particle Penetration in the Full-Face Mask. The assembly, which is composed of the 3D-printed adapter and the filters, was checked for potential leakages before measuring the particle penetration in the complete mask. Because of the limited spatial resolution of the 3D-printing process, the adapter has rough surfaces and microsized gaps can
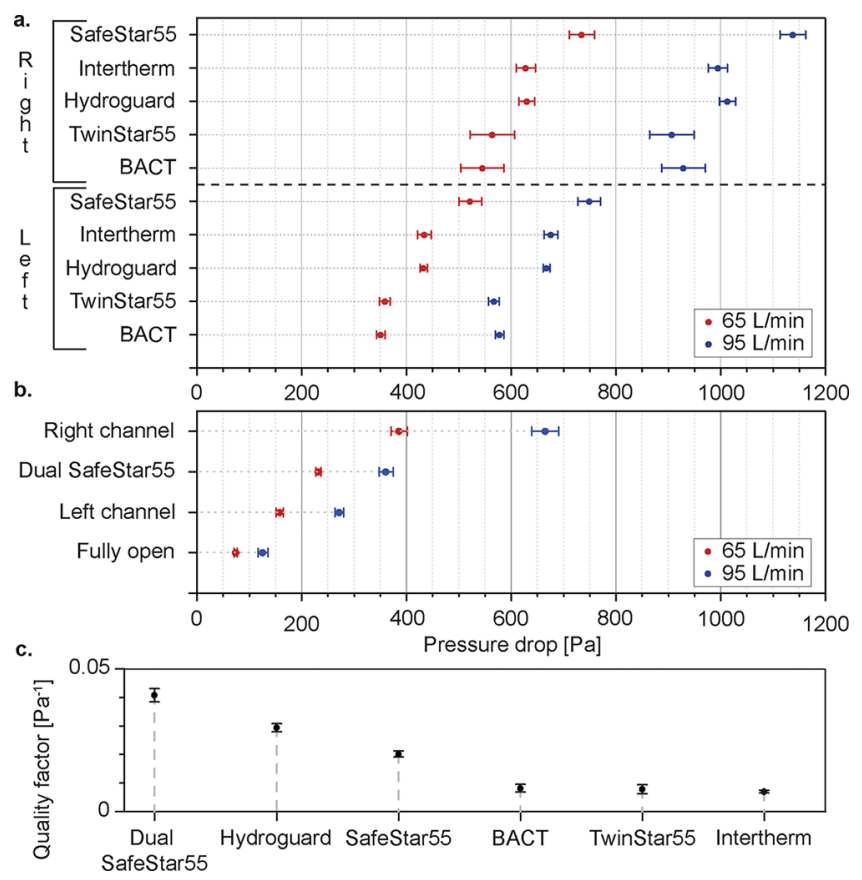

Figure 4. Measurement of the pressure drop and calculation of the quality factor. Panel (a) shows the pressure drop generated by the five medical filters when they were mounted on the face mask with the adapter. The distinction was made between the right and left channel of the adapter (right and left refer to the view shown in Figures $2 \mathrm{f}$ and $2 \mathrm{~g}$ ) to highlight the asymmetry of the flow resistance (the other channel being blocked during the measurement). The measurements were performed at $95 \mathrm{~L} / \mathrm{min}$ to simulate heavy breathing. $65 \mathrm{~L} / \mathrm{min}$ corresponded to the highest flow measured through one filter in the dual-filter configuration (a filter connected to each channel) with a 95 $\mathrm{L} /$ min pumping flow. It takes into account the asymmetry of the flow resistance between both channels. Panel (b) gives the data for the adapters and the dual-filter configuration, as used for the subsequent measurements. Each measurement was repeated five times and the error bars correspond to the standard deviation. In panel (c), the filters were ranked according to their calculated quality factor based on the filtration at MPPS and their pressure drop at $95 \mathrm{~L} / \mathrm{min}$ (after subtracting the pressure drop in each channel). The error bars were calculated by combining the error in the filtration efficiency and in the pressure drop measurements.

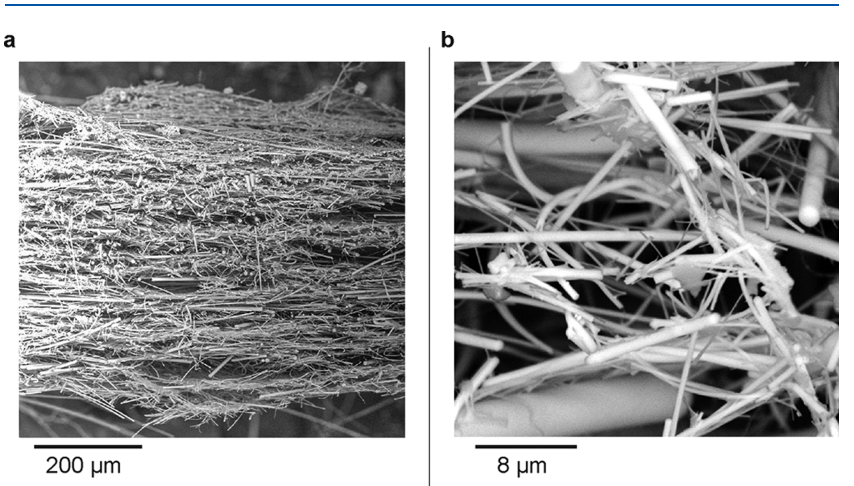

Figure 5. SEM images of the cross-section of a piece of filtering material from the SafeStar55 filter, showing (a) the multiple layers of fibers and (b) the variability in the diameters of the fibers.

form at the interfaces with the filters in the absence of a sealing gasket. However, the measurements showed a particle penetration of $0.2 \%$, which is acceptable. More details are given in Figure S7 in the Supporting Information. On the other 
end of the adapter, at the interface with the mask, the tightness is realized by a sealing gasket located on the port of the mask. The influence of an accumulation of electrostatic charges on the surface of the adapter was not quantified. They interact only with the fraction of the aerosol penetrating through the filters and potentially increase the measured efficiency via the electrostatic removal of particles from the airflow. The charges are likely to appear both in the measurements and in the application. Therefore, the test conditions reflect the real use case.

The SafeStar 55 filter was measured at $95 \mathrm{~L} / \mathrm{min}$ (corresponding to a face velocity of $67 \mathrm{~cm} / \mathrm{s}$ ) to be directly compared with the full-face mask. The setup for the characterization of the filter and the full-face mask in the dual-filter configuration is shown in Figure 6a. The results are presented in Figure $6 \mathrm{~b}$. The increased
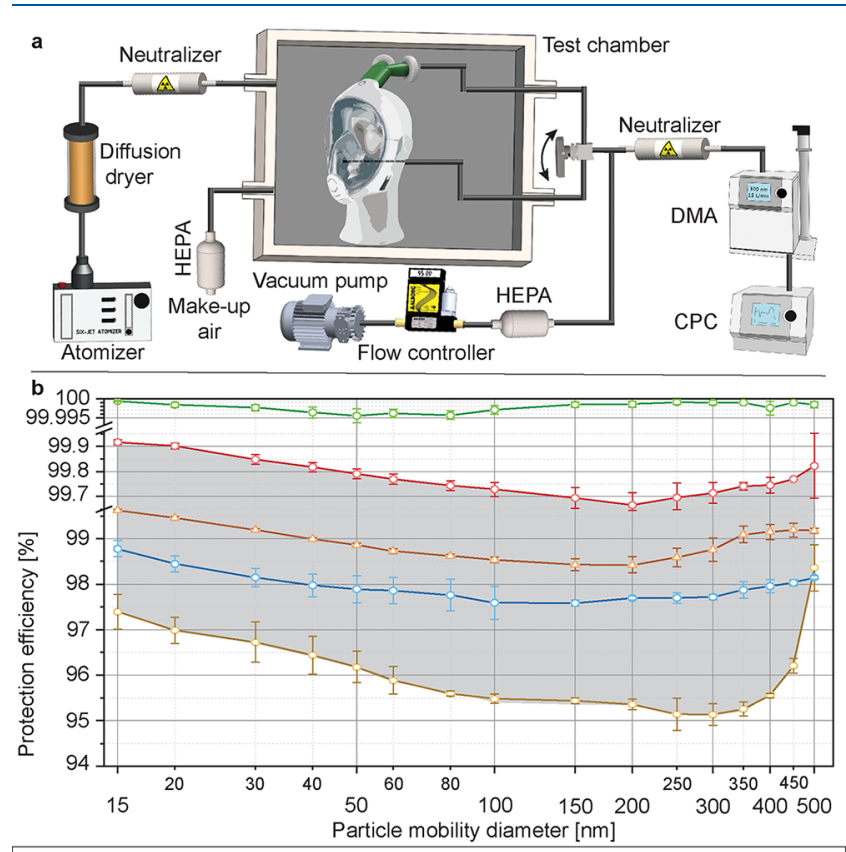

$-\propto-$ Filter SafeStar55 $-\triangle-$ Face mask - Fit Average $-\circ-$ Commercial respirator $-\infty$ Face mask - Fit High - - Face mask - Fit Low

Figure 6. Measurement of the protection efficiency (calculated in the same way as the filtration efficiency and used to characterize the complete mask) of the mask in different fitting configurations. Panel (a) shows the experimental setup, with the full-face mask mounted on a polystyrene dummy head and installed in a stainless steel chamber. Panel (b) presents the results of the protection efficiency against the penetration of $\mathrm{NaCl}$ particles with different mobility diameters at $95 \mathrm{~L} /$ min and different fittings on the dummy head to represent the extreme cases. The gray area represents the range of filtration efficiency, depending on the fitting scenario. It was compared to a commercial fullface respirator (3M Model 6800, equipped with two A2B2E2K2P3 R filters, corresponding to a $\mathrm{P} 3$ particle filter and stopping $99.95 \%$ of the particles, according to the EN 143 standard). The filtration efficiency of the SafeStar55 filter was measured at $95 \mathrm{~L} / \mathrm{min}$. The error bars relate to the standard deviation calculated from three repetitions of 60 measurement points.

airflow led to a decrease of the MPPS of the SafeStar55, compared to the data at $8.5 \mathrm{~cm} / \mathrm{s}$, from $250 \mathrm{~nm}$ to $\sim 50-80 \mathrm{~nm}$. A decrease of the MPPS with increasing face velocity has been previously observed ${ }^{30,31}$ and theoretically explained. ${ }^{32}$ The higher face velocity decreases the efficiency of the diffusiondriven filtration, resulting in a higher penetration for particles $<100 \mathrm{~nm}$ in size. Simultaneously, the higher flow increases the efficiency of the inertial impaction leading to a decrease of the penetration of particles $>100 \mathrm{~nm}$ in size. More details on the influence of the face velocity on the filter are given in Figure S8 in the Supporting Information.

The characterization of the full-face mask was done in the dual-inlet configuration with two SafeStar55 filters. The expression "protection efficiency" is used for the full-face mask to make the distinction with the filtration efficiency characterizing the filters. They are both calculated with the same method.

In order to take into account the variability due to the fitting on the polystyrene head, three different configurations representing the extreme cases were tested:

- The upper curve, marked "High", was the maximal measured filtration efficiency and was obtained after sealing the interface between the polystyrene head and the full-face mask with silicone sealant. This case represents the highest achievable protection efficiency of the face mask and would correspond to a perfect fit of the mask on the wearer's head.

- The lower curve, marked "Low", was measured after the seal was intentionally not fulfilled: the full-face mask was installed on the dummy head but the seal check still showed the presence of leakages, which were deliberately not corrected. The purpose of this scenario is to estimate the lower boundary of the protection efficiency, considering that a fulfilled seal check will lead to a higher protection than the configuration tested in the present case. Even if the exact level of leakage is difficult to reproduce, the protection efficiency is higher with a fulfilled seal check than in this scenario.

- The middle curve marked "Average" is representative of the most frequently measured efficiency. This corresponds to a typical fit on the wearer.

The resulting gray area indicates the protection efficiency range, depending on the quality of the fitting. It encloses an area between $95 \%$ and $99.9 \%$ efficiency. The MPPS was measured $\sim 200 \mathrm{~nm}$ for the best and average cases, increased to $300 \mathrm{~nm}$ for the lower boundary. The efficiency did not go below $98 \%$ when the seal check was meticulously followed. The protection performances of the full-face mask were close to those of the commercial respirator taken as a reference. The measurements highlight the good performances of the full-face mask and the large variability induced by the fitting, and therefore the importance of performing a fitting test before using the mask.

To compare the results obtained on a static polystyrene head with the protection efficiency in realistic operational conditions, the mask with filters (2xSafeStar55) and an adapter was tested, according to the EN 149 standard, requiring movements of the head during the measurements. Three fitting tests were performed with one mask and three volunteers, resulting in particle penetrations of $2.6 \%, 4.8 \%$, and $5.6 \%$ with paraffin particles, fulfilling the requirements for FFP2 masks. The measured values were slightly lower than the values presented in Figure $6 \mathrm{~b}$. This difference can be explained by the test method, since EN 149 requires the wearer to move during the measurements. These movements create small gaps between the skin and the mask and increase the particle penetration. A comparison of the particle penetration in the mask was done with the dummy head, a nonmoving volunteer, and a volunteer reproducing the movements of the head as described by EN 149. The results are given in Figure S9 in the Supporting Information and show a significantly higher particle penetration when the volunteer was moving. 
Decontamination Process. Measuring the impact of decontamination processes on the protection efficiency is critical to assess the reusability of the protective equipment and influences its potential to be successfully used in medical facilities. Autoclaving and immersion into ethanol (70 vol \% ethanol in deionized water) were selected, because of their availability in most medical facilities. The full-face masks were decontaminated without the adapter and filters, since these components are easily replaceable at a reduced cost. The results are presented in Figure 7.

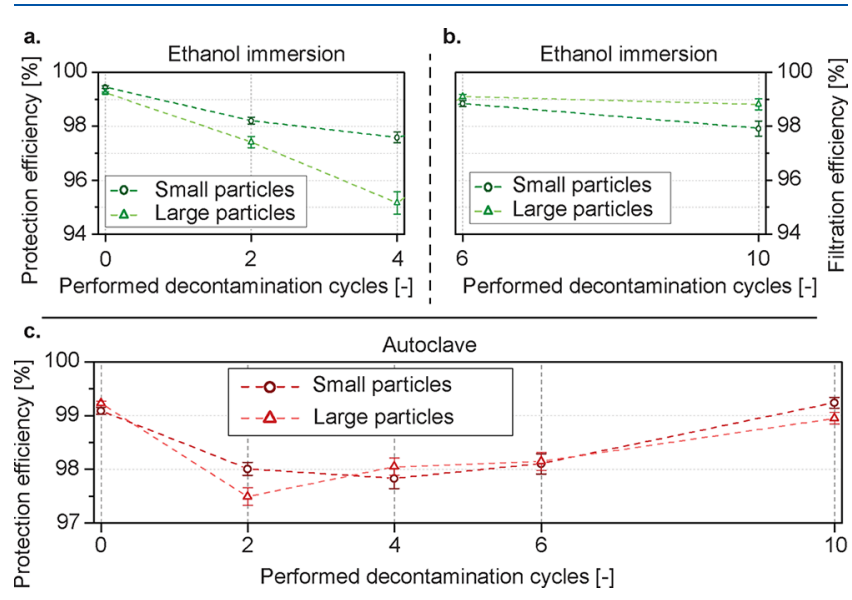

Figure 7. Evolution of the protection efficiency after multiple cycles of decontamination. The sample tested in panels (a) and (b) was immersed in ethanol. The drying time before the measurement was $2 \mathrm{~h}$ at room temperature for panel (a) and was increased to $12 \mathrm{~h}$ in panel (b). Panel (c) displays the protection efficiency of the autoclaved mask. Small particles correspond to a polydisperse aerosol centered at $36 \mathrm{~nm}$, and large particles correspond to a polydisperse aerosol centered at 82 $\mathrm{nm}$.

The immersion into ethanol initially caused a drop of protection efficiency (Figure 7a), more significant for larger particles $(82 \mathrm{~nm}$ peak size) than for smaller particles $(36 \mathrm{~nm}$ peak size). A visual inspection did not reveal any deformations or damages on the mask. Some polymers are subject to swelling through uptake of ethanol after several days of immersion. ${ }^{33,34}$ The face mask is made of different types of polymers: the transparent front cover of the mask is made from acrylonitrile butadiene styrene (ABS) and polycarbonate, the frame is composed of polypropylene and the sealing skirt is made of silicone. A solution of $70 \%$ ethanol has little effect on the mechanical properties of silicone. ${ }^{35}$ Ethanol does not cause significant swelling of polypropylene films, ${ }^{36}$ polycarbonate, ${ }^{37}$ and ABS. ${ }^{38}$ Little information is available on the absorption rates of ethanol, although the rates for water absorption by ABS, polypropylene, and polycarbonate can be used and show little $(<0.002 \%)$ dimensional change after 24 -h exposure. ${ }^{39}$ Another factor can cause the decrease in protection efficiency: it was observed during the measurements that the straps used to fit the mask on the head expanded more when they were still soaked with ethanol after $2 \mathrm{~h}$ of drying. This led to a degraded fitting and therefore to a higher particle penetration. Consequently, the drying time was increased to $12 \mathrm{~h}$ (Figure $7 \mathrm{~b}$ ) for cycle Nos. 6 to 10 and the efficiency recovered close to the starting value. The straps were completely dry $12 \mathrm{~h}$ after being removed from the ethanol solution.

The autoclaved mask (Figure 7c) did not show a significant degradation of its protection performances. The variations can be explained by different drying times between the end of the autoclaving and the measurement. Several damages were detected on the mask: the piece of polymer separating the upper from the lower chamber deformed after two cycles and had to be removed. A gap between the transparent front cover and the frame was also visible, because of the different thermal expansion coefficients of the materials used in both parts. During the removal of the mask from the autoclave after cycle No. 6, the two pieces separated and the mask had to be reassembled manually. Pictures are shown in Figure S10 in the Supporting Information. However, it did not affect the protection efficiency after cooldown.

The decontamination methods did not cause any significant and permanent degradation of the protection efficiency. Nonetheless, autoclaving led to permanent mechanical deformations that can severely impact the particle penetration if the mask is not handled carefully. The masks should be given enough time $(>12 \mathrm{~h})$ to properly dry or to cool down.

\section{CONCLUSION}

This study analyzes the potential of a modified snorkel mask to be used as PPE. First, the filtering efficiency of various medical filters was measured in comparison to FFP2 and FFP3 filter materials. The pressure drop of the medical filters was measured, and the quality factor was calculated. Based on the results, the filter model SafeStar55 was defined as the optimal filter within the framework of this study and used for further testing of the mask. Second, the size-resolved particle penetration in the complete mask was measured. It showed that the mask performed comparable to commercial respirators and the largest source of variability was the fit on the wearer's head. Measurements according to the EN 149 standard showed a compliance with the FFP2 protection level. Lastly, the robustness of the equipment subjected to decontamination cycles with $70 \%$ ethanol and autoclaving was determined. The mask showed little change in protection efficiency over 10 cycles, if enough drying or cooling time was allowed between decontamination and measurement.

The full-face masks described in this work are not advised to replace specifically designed respirators but constitute a decent emergency alternative in the event of a shortage of FFP2 protections, face shields, and full-face respirators to help protecting medical staff exposed to highly contagious patients. This study does not directly demonstrate that these masks reduce the incidence of COVID-19 among healthcare workers, but it shows that they significantly reduce the exposure to potentially contaminated airborne particles and droplets by efficiently covering the face, especially the mouth, the nose, and the eyes. As the infection probability increases through the exposure to a higher viral dose, ${ }^{40}$ this protective equipment can help mitigate the risk of contamination through airborne particles or larger droplets emitted by patients. They only require minor adjustments and are reusable after decontamination without lowering their ability to stop particles. The required adapters are available as an open-source design and can quickly be 3D-printed on site using commonly accessible filaments. The chin valve can be easily and effectively sealed using materials available in most hardware stores.

The mask can be further adapted to better meet the requirements for use in medical facilities: a thinner transparent front cover could reduce the weight and provide better optical properties in order to increase the wearer's comfort. 


\section{ASSOCIATED CONTENT}

\section{(s) Supporting Information}

The Supporting Information is available free of charge at https://pubs.acs.org/doi/10.1021/acs.chemrestox.0c00371.

Setup for the measurement of the pressure drop; photograph of the filters; tested methods to seal the chin valve; size distribution of the particles generated by the 1 and 0.01 wt $\% \mathrm{NaCl}$ solutions; influence of the chin valve on the particle penetration; SEM images of the SafeStar55 filtering material; evaluation of the adapter assembly; evolution of the filtration efficiency of the SafeStar55 with increasing flow rate; dispersion of the filtration efficiency for the volunteer and the dummy head; photographs of the damaged mask after autoclaving (PDF)

\section{AUTHOR INFORMATION}

\section{Corresponding Author}

Jing Wang - Institute of Environmental Engineering, Department of Civil, Environmental and Geomatic Engineering, ETH Zurich, Zurich 8093, Switzerland; Laboratory for Advanced Analytical Technologies, EMPA, Swiss Federal Laboratories for Materials Science and Technology, Dubendorf 8600, Switzerland; 이잉.org/ 0000-0003-2078-137X; Email: jing.wang@ifu.baug.ethz.ch

\section{Authors}

Jean Schmitt - Institute of Environmental Engineering, Department of Civil, Environmental and Geomatic Engineering, ETH Zurich, Zurich 8093, Switzerland; Laboratory for Advanced Analytical Technologies, EMPA, Swiss Federal Laboratories for Materials Science and Technology, Dubendorf 8600, Switzerland

Lewis S. Jones - Institute for Chemical- and Bioengineering, Department of Chemistry and Applied Biosciences, ETH Zurich, Zurich 8093, Switzerland

Elise A. Aeby - Laboratory of Applied Mechanobiology, Department of Health Sciences and Technology, ETH Zurich, Zurich 8093, Switzerland

Christian Gloor - Spiez Laboratory Division CBRNe Protection Systems, Federal Office of Civil Protection, Spiez 3700, Switzerland

Berthold Moser - Department of Anesthesia and Intensive Care, Medical University of Innsbruck, Innsbruck 6020, Austria

Complete contact information is available at:

https://pubs.acs.org/10.1021/acs.chemrestox.0c00371

\section{Author Contributions}

J.S. and J.W. conceived the testing plan. L.J. led the modification of the face mask, designed and fabricated the adapters, and provided the masks and the filters. E.A. defined the sealing method for the chin valve. J.S. conducted the measurements in J.W.'s group, as well as the data analysis, J.S. and E.A. contributed to the comparison of the filters. C.G. performed the measurements according to the EN 149 standard, B.M. tested the mask in medical facilities and gave his feedback. All authors have contributed to the manuscript and have given approval to the final version of the manuscript. All of the photographs appearing in the figures were taken by the authors.

Notes

The authors declare no competing financial interest.

\section{ACKNOWLEDGMENTS}

The authors would like to thank the initiative helpful ETH that brought the team together and D. Kalinic for his contribution to the testing of the face mask. The work was partially supported by Innosuisse project 46668.1 IP-ENG "ReMask: Strategies for innovations for Swiss masks needed in pandemic situations”.

\section{ABBREVIATIONS}

PPE, personal protective equipment; CPC, condensation particle counter; SMPS, scanning mobility particle sizer; MPPS, most penetrating particle size; DMA, differential mobility analyzer; HEPA, high-efficiency particulate air; ABS, acrylonitrile butadiene styrene; PMMA, poly(methyl methacrylate); HME, heat and moisture exchanger; SEM, scanning electron microscope

\section{REFERENCES}

(1) Ranney, M. L., Griffeth, V., and Jha, A. K. (2020) Critical Supply Shortages-The Need for Ventilators and Personal Protective Equipment during the Covid-19 Pandemic. N. Engl. J. Med. 382, e41.

(2) Leung, W. W. F., and Sun, Q. (2020) Electrostatic charged nanofiber filter for filtering airborne novel coronavirus (COVID-19) and nano-aerosols. Sep. Purif. Technol. 250, 116886.

(3) Liu, D. C. Y., Koo, T. H., Wong, J. K. K., Wong, Y. H., Fung, K. S. C., Chan, Y., and Lim, H. S. (2020) Adapting Reusable Elastomeric Respirators to Utilise Anaesthesia Circuit Filters Using a 3D-Printed Adaptor-A Potential Alternative to Address N95 Shortages During the COVID-19 Pandemic. Anaesthesia 75, 1022.

(4) Kroo, L., Kothari, A., Hannebelle, M., Herring, G., Pollina, T., Chang, R., Peralta, D., Banavar, S. P., Flaum, E., and Soto-Montoya, H., et al. Pneumask: Modified Full-Face Snorkel Masks as Reusable Personal Protective Equipment for Hospital Personnel, medRxiv preprint, published online April 24, 2020 DOI: 10.1101/ 2020.04.24.20078907.

(5) Kechli, M. K., Lerman, J., and Ross, M. M. (2020) Modifying a Full-Face Snorkel Mask to Meet N95 Respirator Standards for Use With Coronavirus Disease 2019 Patients. A\&A Practice 14, e01237.

(6) Zhu, N., Zhang, D., Wang, W., Li, X., Yang, B., Song, J., Zhao, X., Huang, B., Shi, W., Lu, R., et al. (2020) A Novel Coronavirus from Patients with Pneumonia in China, 2019. N. Engl. J. Med. 382, 727.

(7) Liu, Y., Ning, Z., Chen, Y., Guo, M., Liu, Y., Gali, N. K., Sun, L., Duan, Y., Cai, J., Westerdahl, D., et al. (2020) Aerodynamic Analysis of SARS-CoV-2 in Two Wuhan Hospitals. Nature 582, 557-560.

(8) Wei, J., and Li, Y. (2015) Enhanced spread of expiratory droplets by turbulence in a cough jet. Building and Environment 93, 86-96.

(9) Morawska, L., Johnson, G. R., Ristovski, Z. D., Hargreaves, M., Mengersen, K., Corbett, S., Chao, C. Y. H., Li, Y., and Katoshevski, D. (2009) Size Distribution and Sites of Origin of Droplets Expelled From the Human Respiratory Tract During Expiratory Activities. J. Aerosol Sci. 40, 256-269.

(10) Jayaweera, M., Perera, H., Gunawardana, B., and Manatunge, J. (2020) Transmission of COVID-19 Virus by Droplets and Aerosols: A Critical Review on the Unresolved Dichotomy. Environ. Res. 188, 109819.

(11) Anderson, E. L., Turnham, P., Griffin, J. R., and Clarke, C. C. (2020) Consideration of the Aerosol Transmission for COVID-19 and Public Health. Risk Anal. 40, 902-907.

(12) Wang, J., and Tronville, P. (2014) Toward Standardized Test Methods to Determine the Effectiveness of Filtration Media Against Airborne Nanoparticles. J. Nanopart. Res. 16, 2417.

(13) Judson, S. D., and Munster, V. J. (2019) Nosocomial Transmission of Emerging Viruses via Aerosol-Generating Medical Procedures. Viruses 11, 940.

(14) He, W., Zhao, Y.-B., Jiang, F., Guo, Y., Gao, H., Liu, J., and Wang, J. (2020) Filtration Performance and Charge Degradation During Particle Loading and Reusability of Charged PTFE Needle Felt Filters. Sep. Purif. Technol. 233, 116003. 
(15) Sun, Z., Liang, Y., He, W., Jiang, F., Song, Q., Tang, M., and Wang, J. (2019) Filtration Performance and Loading Capacity of NanoStructured Composite Filter Media for Applications with High Soot Concentrations. Sep. Purif. Technol. 221, 175-182.

(16) Yin-Murphy, M., and Almond, J. W.. (1996) Picornaviruses. In Medical Microbiology. 4th Edition; Baron, S, Ed.; The University of Texas Medical Branch at Galveston, Chapter 53.

(17) Pattison, J. R., and Patou, G. (1996) Parvoviruses. In Medical Microbiology. 4th Edition; Baron, S., Ed.; The University of Texas Medical Branch at Galveston, Chapter 64.

(18) Sachinidou, P., Bahk, Y. K., and Wang, J. (2017) An Integrative Model for the Filtration Efficiencies in Realistic Tests with Consideration of the Filtration Velocity Profile and Challenging Particle Size Distribution. Aerosol Sci. Technol. 51 (2), 178-187.

(19) 3M Personal Safety Division. Full Facepiece Respirator 6000 Series - User Instructions, Downloaded from https://multimedia.3m.com/ mws/media/959950/3m-6000-series-full-facepiece-respirator-userinstructions.pdf on July 3rd, 2020.

(20) Wang, J., Kim, S. C., and Pui, D. Y. H. (2008) Investigation of the Figure of Merit for Filters with a Single Nanofiber Layer on a Substrate. J. Aerosol Sci. 39, 323-334.

(21) Wang, J., Kim, S. C., and Pui, D. Y. H. (2008) Figure of Merit of Composite Filters with Micrometer and Nanometer Fibers. Aerosol Sci. Technol. 42, 722-728.

(22) Huang, S.-H., Chen, C.-W., Kuo, Y.-M., Lai, C.-Y., McKay, R., and Chen, C.-C. (2013) Factors Affecting Filter Penetration and Quality Factor of Particulate Respirators. Aerosol Air Qual. Res. 13, $162-171$.

(23) Quanjer, P. H., Tammeling, G. J., Cotes, J. E., Pedersen, O. F., Peslin, R., and Yernault, J.-C. (1993) Lung Volumes and Forced Ventilatory Flows. Eur. Respir. J. 6, 5-40.

(24) Hulkower, R. L., Casanova, L. M., Rutala, W. A., Weber, D. J., and Sobsey, M. D. (2011) Inactivation of Surrogate Coronaviruses on Hard Surfaces by Health Care Germicides. Am. J. Infect. Control 39, 401-407.

(25) Chin, A. W H, Chu, J. T S, Perera, M. R A, Hui, K. P Y, Yen, H.-L., Chan, M. C W, Peiris, M., and Poon, L. L M (2020) Stability of SARSCoV-2 in Different Environmental Conditions. Lancet Microbe 1, e10.

(26) Liao, L., Xiao, W., Zhao, M., Yu, X., Wang, H., Wang, Q., Chu, S., and Cui, Y. (2020) Can N95 Respirators Be Reused after Disinfection? How Many Times? ACS Nano 14, 6348-6356.

(27) Lin, T.-H., Chen, C.-C., Huang, S.-H., Kuo, C.-W., Lai, C.-Y., and Lin, W.-Y. (2017) Filter Quality of Electret Masks in Filtering 14.6$594 \mathrm{~nm}$ Aerosol Particles: Effects of Five Decontamination Methods. PLoS One 12, e0186217.

(28) Li, Y., Guo, Y. P., Wong, K. C. T., Chung, W. Y. J., Gohel, M. D. I., and Leung, H. M. P. (2008) Transmission of Communicable Respiratory Infections and Facemasks. JMDH 2008, 17-27.

(29) Serfozo, N., Ondracek, J., Zikova, N., Lazaridis, M., and Zdimal, V. (2017) Size-Resolved Penetration of Filtering Materials from CEMarked Filtering Facepiece Respirators. Aerosol Air Qual. Res. 17, $1305-1315$.

(30) Liu, J., Pui, D. Y. H., and Wang, J. (2011) Removal of Airborne Nanoparticles by Membrane Coated Filters. Sci. Total Environ. 409, $4868-4874$

(31) Al-Attar, I. S., Wakeman, R. J., Tarleton, E. S., and Husain, A. (2011) The Effect of Face Velocity, Pleat Density and Pleat Orientation on the Most Penetrating Particle Size, Pressure Drop and Fractional Efficiency of HEPA Filters. In Proceedings of the Filtech Conference; Filtech Exhibitions, Wiesbaden, Germany; pp 318-329.

(32) Lee, K. W., and Liu, B. Y. H. (1980) On the Minimum Efficiency and the Most Penetrating Particle Size for Fibrous Filters. J. Air Pollut. Control Assoc. 30, 377-381.

(33) Chuang, W.-Y., Young, T.-H., Wang, D.-M., Luo, R.-L., and Sun, Y.-M. (2000) Swelling Behavior of Hydrophobic Polymers in Water/ Ethanol Mixtures. Polymer 41, 8339-8347.

(34) Sachinidou, P., Heuschling, C., Schaniel, J., and Wang, J. (2018) Investigation of Surface Potential Discharge Mechanism and Kinetics in Dielectrics Exposed to Different Organic Solvents. Polymer 145, 447453.
(35) Crnich, C. J., Halfmann, J. A., Crone, W. C., and Maki, D. G. (2005) The Effects of Prolonged Ethanol Exposure on the Mechanical Properties of Polyurethane and Silicone Catheters Used for Intravascular Access. Infection Control and Hospital Epidemiology 26, 708714.

(36) Barson, C. A., and Dong, Y. M. (1990) Diffusion and Swelling Studies of Ethanol and Ethyl Laurate with Dry Polypropylene and Polyethylene Terephthalate Films Using a Radiotracer Technique. Eur. Polym. J. 26, 449-451.

(37) Thermoscientific. Labware chemical resistance table, http://tools. thermofisher.com/content/sfs/brochures/D20480.pdf, consulted on July 11, 2020.

(38) Millipore, Chemical Compatibility of Filter Components, https://www.emdmillipore.com/Web-CA-Site/en_CA/-/CAD/ Show Document-Pronet ? id = $201510 . \overline{3} 99 \&$ u s g = AOvVaw3h0KMcgRcLW-ZMsoV9AlbV, accessed on July 11, 2020.

(39) Mitsubishi Engineering Plastics Corporation. Rate of Water and Moisture Absorption, https://www.m-ep.co.jp/en/pdf/product/iupi nova/physicality 06.pdf, viewed on July 11, 2020.

(40) Zhang, X., and Wang, J. (2020) Dose-response Relation Deduced for Coronaviruses from COVID-19, SARS and MERS Meta-analysis Results and its Application for Infection Risk Assessment of Aerosol Transmission. Clin. Infect. Dis., ciaa 1675. 